\title{
Epstein-Barr virus-positive lymphoproliferative disorder manifesting as pulmonary disease in a patient with acute myeloid leukemia: a case report
}

\author{
Ritika Dutta ${ }^{1,2}$, Susanna Y. Miao ${ }^{3}$, Paul Phan ${ }^{1}$, Sebastian Fernandez-Pol ${ }^{4}$, Parveen Shiraz ${ }^{5}$, Dora Ho ${ }^{6}$,
} Gabriel N. Mannis ${ }^{1}$ and Tian Y. Zhang ${ }^{1,2^{*}}$

\begin{abstract}
Background: Patients with lymphoproliferative disorders following hematopoietic stem cell transplant (HSCT) most commonly present with fever and lymphadenopathy within the first 5 months of transplant. Pulmonary post-transplant lymphoproliferative disorder (PTLD) is a particularly aggressive and rapidly progressive disease, with high morbidity and mortality. There are a very limited number of reported pulmonary PTLD cases following HSCT in patients with acute myeloid leukemia (AML). Early diagnosis and detection of pulmonary PTLD is critical given its high lethality. However, variable clinical presentations and nonspecific radiographic findings make pulmonary PTLD difficult to distinguish from other more common causes of pulmonary disease in AML patients.
\end{abstract}

Case presentation: Here, we describe a 68-year-old Caucasian man who presented for salvage induction therapy following relapse of his AML after a haploidentical allogeneic HSCT 10 months earlier. He developed recurrent fevers, dry cough, and hypoxemia, with chest computed tomography (CT) showing bibasilar consolidations and increased nodularity without increased lymphadenopathy. His symptoms initially improved with antibiotic and antifungal therapy, but his follow-up chest CT showed progression of disease despite symptomatic improvement. Epstein-Barr virus (EBV) was detected in his blood by polymerase chain reaction (PCR), and a lung biopsy revealed monomorphic PTLD with B cells positive for EBV. Unfortunately, the patient's condition rapidly deteriorated, and he passed away prior to treatment initiation.

Conclusions: To our knowledge, this is the first reported case of an AML patient developing pulmonary PTLD relatively late in his post-transplant course in the setting of relapsed disease and salvage therapy. Pulmonary PTLD, a rare but highly lethal disorder, can imitate the symptoms and radiographic findings of pneumonia, a common diagnosis in immunocompromised AML patients. This case illustrates the importance of considering pulmonary PTLD in the differential diagnosis for pulmonary disease in AML patients with a history of HSCT, especially in the setting of progressive radiographic findings despite broad antibacterial and antifungal therapy. Further, our case demonstrates the importance of biopsy and uninterrupted EBV DNA monitoring in the definitive diagnosis of PTLD, given nonspecific symptomatology and radiographic findings.

\footnotetext{
*Correspondence: tzhang8@stanford.edu

1 Department of Medicine, Division of Hematology, Cancer Institute,

and Institute for Stem Cell Biology and Regenerative Medicine, Stanford

University, Stanford, CA, USA

Full list of author information is available at the end of the article
} permits use, sharing, adaptation, distribution and reproduction in any medium or format, as long as you give appropriate credit to the original author(s) and the source, provide a link to the Creative Commons licence, and indicate if changes were made. The images or other third party material in this article are included in the article's Creative Commons licence, unless indicated otherwise in a credit line to the material. If material is not included in the article's Creative Commons licence and your intended use is not permitted by statutory regulation or exceeds the permitted use, you will need to obtain permission directly from the copyright holder. To view a copy of this licence, visit http://creativecommons.org/licenses/by/4.0/. The Creative Commons Public Domain Dedication waiver (http://creativeco mmons.org/publicdomain/zero/1.0/) applies to the data made available in this article, unless otherwise stated in a credit line to the data. 
Keywords: Acute myeloid leukemia, Epstein-Barr virus, Post-transplant lymphoproliferative disorder, Hematopoietic stem cell transplant, Case report

\section{Background}

Epstein-Barr virus-associated post-transplant lymphoproliferative disorders (EBV-PTLDs) are a heterogenous group of rare, but potentially fatal, diseases that can develop following allogeneic hematopoietic stem cell transplant (allo-HSCT) or solid-organ transplant (SOT) $[1,2]$. De novo EBV infection or EBV reactivation in the setting of compromised T-cell immunity can lead to transformation and immortalization of lymphocytes, most commonly B cells, subsequently resulting in uncontrolled proliferation. Compared with SOT, postHSCT PTLD has a more aggressive clinical course, inferior response to therapy, and shortened overall survival [3]. The overall incidence of PTLD in allo-HSCT recipients ranges from 0.5 to $6 \%$ and is particularly high in the pediatric population [1, 4-9]. Most of these cases are EBV-related, donor-derived, and develop within 1 year of HSCT, with the highest incidence occurring in the first 5 months following transplant $[1,5]$. Known risk factors for PTLD include several transplant-associated characteristics, including reduced-intensity conditioning, T-cell depletion of the graft, inclusion of anti-thymocyte globulin (ATG) for graft-versus-host disease (GVHD) prophylaxis, mismatch of human leukocyte antigens, and severe GVHD [5, 6].

Clinically, patients with EBV-PTLD most commonly present with fever, lymphadenopathy, and/or infectious mononucleosis symptoms. Patients can also develop extranodal disease, most commonly in the liver, gastrointestinal tract, spleen, central nervous system, and lungs $[1,3,9]$. Early detection of EBV in the blood by polymerase chain reaction (PCR) has greatly improved outcomes for EBV-PTLD, allowing for timely treatment with rituximab. Guidelines from the European Conference on Infections in Leukaemia recommend weekly screening for EBV-DNA in the blood (EBV DNAemia) for at least 4 months following transplant [10]. The time from initial detection of EBV DNAemia to clinical manifestations of EBV-associated disease is typically very short, with a median of 7 days, highlighting the rapidly progressive nature of the disease [11]. Despite appropriate treatment, mortality for EBV-PTLD remains approximately 30\% [10].

Pulmonary PTLD following allo-HSCT is a poorly understood and particularly aggressive form of the disease, with a median reported duration between diagnosis and death of 5 days [7]. Thus, early clinical suspicion and detection is crucial to improve survival. However, this remains difficult because pulmonary PTLD is scarcely described in medical literature and has a wide range of presentations, from localized disease to disseminated lymphoma with multiorgan dysfunction [7]. From our review of the literature, there have been few reports of pulmonary PTLD cases arising in patients with adult acute myeloid leukemia (AML) following allo-HSCT [7, 12]. Most reported cases occurred in younger patients $(<40)$ with known risk factors, such as severe GVHD and ATG immunosuppression, and presented within 2 months of transplant. Here, we report a unique case of a 68-year-old man who developed pulmonary PTLD 10 months following his allo-HSCT while receiving salvage therapy for relapsed AML.

\section{Case presentation}

A 68-year-old Caucasian man with a past medical history of Crohn's disease, hypertension, type 2 diabetes mellitus, obstructive sleep apnea, and atrial fibrillation, as well as noncontributory psychosocial or family history, was diagnosed with adverse risk, therapy-related AML after presenting with progressive cytopenias (Fig. 1). His bone marrow (BM) biopsy was remarkable for $27 \%$ blasts in a background of trilineage dysplasia with a karyotype of $\operatorname{del}(5 q), \operatorname{del}(20 q)$, and trisomy 8 . Targeted next-generation sequencing revealed a pathogenic somatic mutation in TP53 as well as variants of unknown significance in ASXL1, FAS, and PIK3CA. AML with myelodysplasiarelated changes was diagnosed based on his TP53 mutation and complex karyotype. He achieved first complete remission (CR1) with no measurable residual disease (minimal residual disease [MRD]-negative) by multiparametric flow cytometry after 5 cycles of decitabine/venetoclax combination therapy. His initial chemotherapy course was complicated by the development of pulmonary nodules within 1 month of treatment initiation, concerning for invasive fungal infection (without microbiologic confirmation). He was treated with posaconazole with subsequent resolution. He then underwent haploidentical HSCT with peripheral blood stem cells from a relative using a reduced-intensity conditioning regimen consisting of fludarabine, cyclophosphamide, and totalbody irradiation (Flu/CY/TBI). GVHD prophylaxis consisted of post-transplant cyclophosphamide, tacrolimus, and mycophenolate mofetil. His post-transplant course was complicated by Morganella morganii bacteremia, a Demodex skin infection, and grade II-III acute GVHD of the skin. Peripheral blood chimerism on day +90 


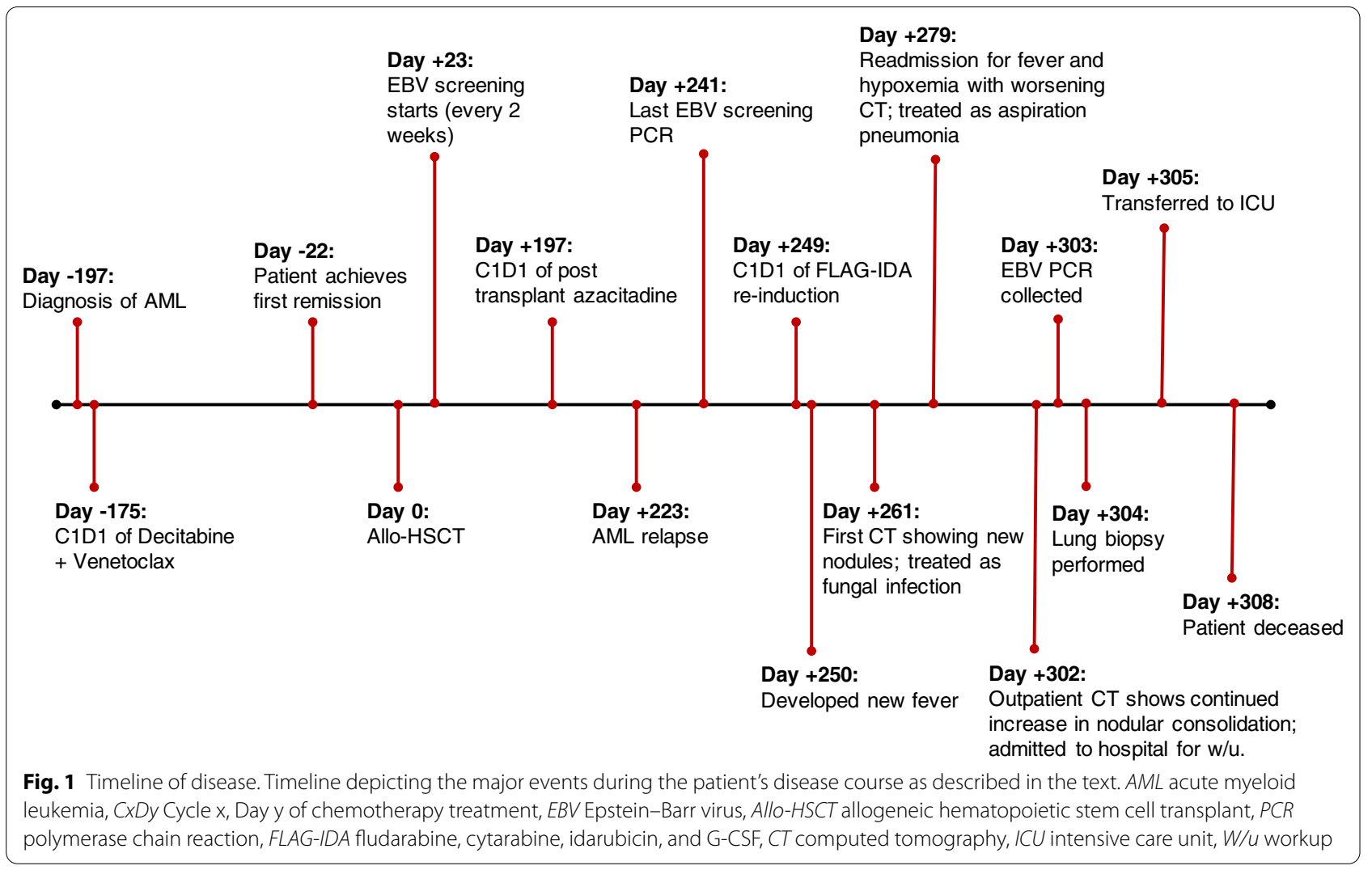

post-transplant demonstrated successful engraftment, with $99-100 \%$ donor cells, and a BM biopsy showed continued MRD-negative CR.

Unfortunately, a repeat BM biopsy 6 months following transplant showed a $0.2 \%$ abnormal blast population by flow cytometry. To augment the graft-versus-leukemia effect, the patient received two cycles of post-allo-HSCT azacitidine, and immunosuppressants were rapidly tapered. However, a BM biopsy on day +223 following transplant revealed relapsed AML with a $10 \%$ blast population exhibiting complex cytogenetics and a pathogenic mutation in TP53, as well as variants of unknown significance in FAS, PIK3CA, SETD2, and TET2, with mixed chimerism. The patient was then admitted to the hospital for salvage induction with FLAG-IDA (fludarabine, cytarabine, idarubicin, and granulocyte colony-stimulating factor [G-CSF]).

During the hospital admission for FLAG-IDA induction (day +249 post-transplant), the patient developed febrile neutropenia with throat irritation and an accompanying cough. His physical exam was notable for decreased breath sounds and bilateral wheezes. He was found to have respiratory syncytial virus and started on ribavirin (oral) with meropenem and levofloxacin due to persistent fevers and a new oxygen requirement. A chest computed tomography (CT) scan (day +261) scan demonstrated new nodular opacities with surrounding ground glass along with bibasilar consolidations (Fig. 2a, b). Due to concern for possible pulmonary fungal infection, bronchoscopy was performed. Bronchoalveolar lavage (BAL) was only remarkable for an Aspergillus galactomannan index $>2$; serum cell-free fungal DNA was negative. Given radiographic findings suspicious for a fungal infection and a positive BAL fungal marker, antifungal treatment was started using intravenous liposomal amphotericin B (AmBisome $\left.{ }^{\circledR}\right)$. His fever curve and oxygen requirement improved after initiation of antifungal therapy and count recovery. He was then discharged on posaconazole, as well as prophylactic acyclovir and trimethoprim/sulfamethoxazole. However, he was readmitted shortly afterwards with low-grade fever, rigors, chills, cough, and hypoxemia. A chest/abdomen/pelvis CT scan $($ day +279$)$ was remarkable for increased confluence of consolidations in both lower lobes and additional nodules throughout both lungs (Fig. 2a). The patient was treated for a presumed aspiration pneumonia with broad-spectrum antibiotics, which led to resolution of the fevers and improvement of the cough.

Two weeks following discharge, outpatient CT chest $($ day +302$)$ imaging revealed progressive bronchocentric 


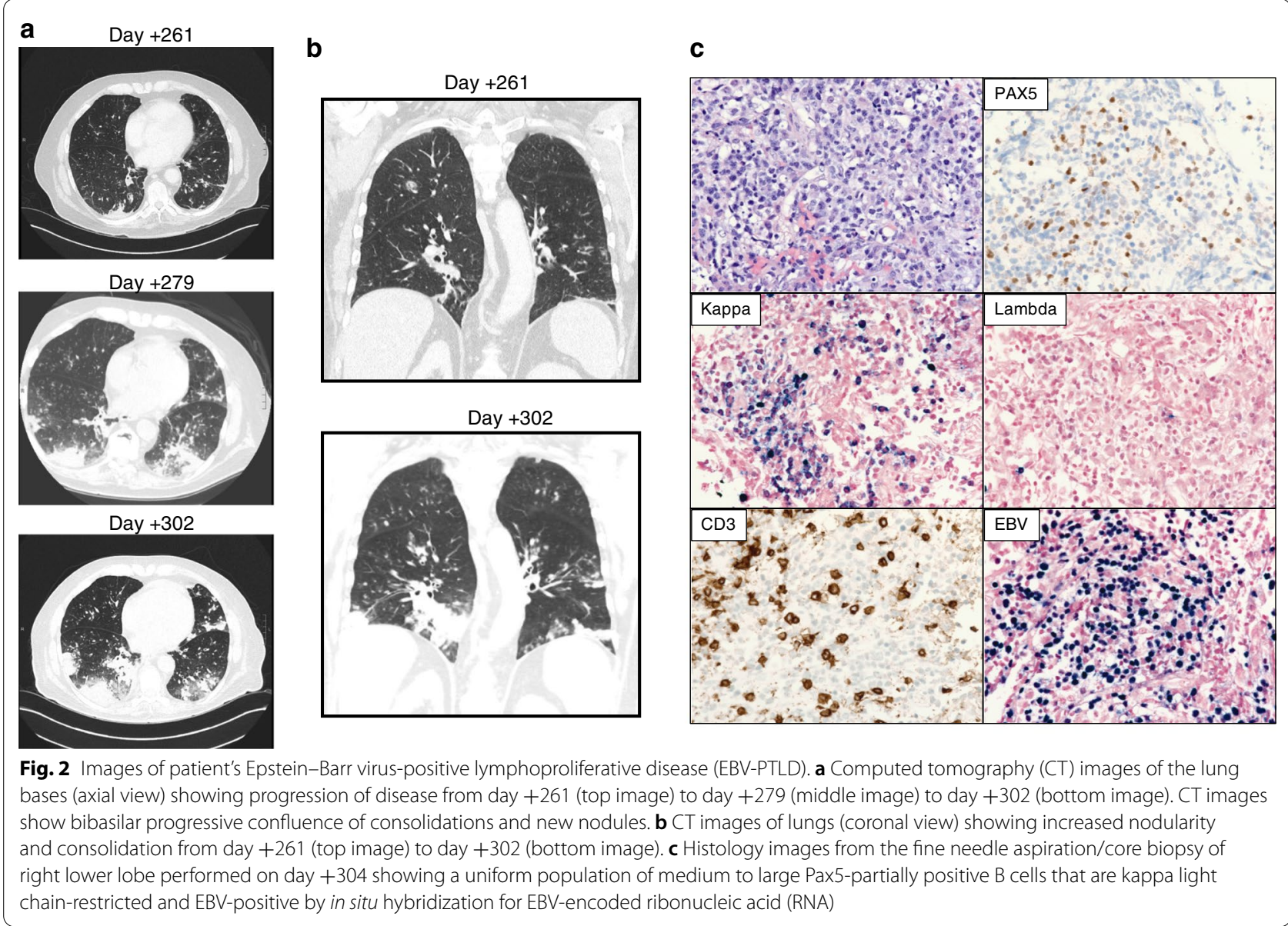

and basilar-predominant nodular consolidations despite continued treatment with posaconazole (Fig. 2a, b). Additionally, the patient continued to experience a persistent cough with clear sputum and without associated dyspnea or B symptoms. He was readmitted to the hospital for expedited workup of the CT findings, with concern for a persistent invasive fungal infection. EBV-PCR was ordered (day +303), and fine needle aspiration and core biopsy of the right lower lobe were performed (day $+304)$. Unfortunately, the patient developed increased work of breathing and altered mental status, necessitating transfer to the intensive care unit (ICU) (day +305).

His EBV deoxyribonucleic acid polymerase chain reaction (DNA PCR) results returned positive on day +308 at $23,142 \mathrm{IU} / \mathrm{mL}$. Of note, the patient was screened every 2 weeks for EBV-DNA from day +23 post-transplant to day +241 with negative results. The lung biopsy revealed a uniform population of medium to large lymphoid cells with irregular nuclear contours, coarse chromatin, and scant cytoplasm. The final diagnosis was monomorphic PTLD with EBV-positive diffuse large B-cell lymphoma (Fig. 2c). By flow cytometry, the abnormal B-cell population expressed CD38 (bright), CD45, surface CD19, surface CD22 (77\%), and CD20 (62\%), and was negative for CD5, CD10, and surface light chain expression. In situ hybridization showed that the PTLD was EBV-positive and kappa light chain-restricted (Fig. 2c). A periodic acid-Schiff-diastase stain did not demonstrate any fungal organisms. Although preliminary results from the EBV DNA and lung biopsy became available on day +308 , the patient's condition had acutely deteriorated, requiring intubation and pressor support. Based on his family's wishes, he was transitioned to comfort care and passed away on day +308 .

\section{Discussion and conclusions}

Pulmonary disease in a relapsed AML patient with a history of allo-HSCT has a broad differential, including infectious or noninfectious causes of pneumonia/ pneumonitis, GVHD, and PTLD [13]. Symptomatology and imaging findings can be nonspecific and can overlap among these diagnoses. Typically, pulmonary PTLD is donor-derived, and patients present with fever, dyspnea and hypoxemia, diffuse lymphadenopathy, and systemic 
symptoms within 1-5 months following allo-HSCT [7, 13]. Radiographic features can include diffuse infiltrates in the basal and subpleural areas, pulmonary nodules, cavitation, peribronchial thickening, and reticulonodular shadowing [7, 13-15]. We describe a case of a 68-yearold man who developed pulmonary PTLD relatively late in his post-transplant course after tapering of immunosuppressive agents in the setting of AML salvage therapy with FLAG-IDA. He presented with fevers and a dry cough and later developed systemic symptoms. Although his symptoms were recurrent, they initially improved with antibiotic and antifungal therapy during each hospitalization, suggesting the possibility of a concomitant infectious process. He also did not demonstrate any new lymphadenopathy by physical exam or CT imaging during progression of his PTLD. Combined with his neutropenia and CT findings of bibasilar consolidations and increased nodularity, his clinical presentation was initially concerning for a pneumonia. Clinical suspicion for PTLD should be heightened in patients who have progressive radiographic pulmonary disease despite appropriate antifungal and antibacterial treatment. EBV-PCR can then be obtained to assess for EBV DNAemia as a marker for PTLD. Of note, our patient developed EBV DNAemia in the 62-day window between his last negative screen on day +241 post-transplant and the positive sample tested on day +303 . It is possible that the new pulmonary nodules seen on his CT scan on day +261 represented the initial PTLD lesions, but this cannot be confirmed with certainty. Interestingly, our patient developed PTLD in the setting of FLAG-IDA salvage therapy after weaning of his transplant immunosuppressive regimen. This raises the possibility that chemotherapy may have contributed to his immunosuppressed state and development of lymphoproliferative disease [16]. Consistent with prior reports, this case reinforces the recommendations for frequent screening given the rapid clinical course of EBV-PTLD after the initial detection of EBV DNAemia $[1,7,9]$. Furthermore, given the variable findings of PTLD on radiographic imaging, this case highlights the importance of obtaining a lung biopsy for definitive diagnosis of PTLD to ensure that appropriate therapy can be initiated in a timely manner [2]. Although rituximab has been shown to be an efficacious therapy for EBV-PTLD, mortality in pulmonary PTLD patients remains high despite treatment, underlining a need for further research into the pathogenesis and therapeutic approaches for pulmonary PTLD. Newly available therapies that target other antigens such as CD19 (blinatumomab) and CD22 (inotuzumab) warrant study to see whether outcomes can be improved.

In conclusion, this case provides important anecdotal evidence to support the inclusion of pulmonary PTLD in the differential diagnosis of pulmonary disease in AML patients with a prior history of allo-HSCT. To our knowledge, this is the first reported case of an AML patient developing pulmonary PTLD relatively late in their posttransplant course in the setting of relapsed disease and salvage therapy. Our case highlights the importance of biopsy and frequent EBV DNA monitoring in the definitive diagnosis of PTLD, given nonspecific symptomatology and radiographic findings that can imitate other causes of pulmonary disease in AML patients.

\section{Abbreviations \\ Allo-HSCT: Allogeneic hematopoietic stem cell transplant; AML: Acute myeloid leukemia; ATG: Anti-thymocyte globulin; BAL: Bronchoalveolar lavage; BM: Bone marrow; CR: Complete remission; CT: Computed tomography; EBV: Epstein-Barr virus; EBV-PTLD: Epstein-Barr virus-associated post-transplant lymphoproliferative disorder; FLAG-IDA: Fludarabine, cytarabine, idarubicin, and granulocyte colony-stimulating factor (G-CSF); GVHD: Graft-versus-host disease; MRD: Minimal residual disease; PCR: Polymerase chain reaction; PTLD: Post-transplant lymphoproliferative disease/disorder; SOT: Solid organ transplant.}

\section{Acknowledgements}

This manuscript was written in loving memory of the patient. We thank and acknowledge his family for their support. We would also like to acknowledge our fellow healthcare workers who took part in caring for the patient.

\section{Authors' contributions}

$\mathrm{RD}$ participated in patient care, compiled the data, and wrote the manuscript. SFP provided pathology images and edited the manuscript. SYM, PS, DH, and GNM took part in patient care and manuscript editing. TZ was the primary caregiver for the patient and contributed to manuscript writing and editing along with PP.

\section{Funding}

The authors declare no source of funding for this study.

\section{Availability of data and materials}

Data sharing is not applicable as no data sets were generated or analyzed during the study.

\section{Ethics approval and consent to participate}

Not applicable.

\section{Consent for publication}

Written informed consent was obtained from the patient's family for publication of this case report and any accompanying images. A copy of the written consent is available for review by the Editor-in-Chief of this journal.

\section{Competing interests}

The authors declare no competing interests.

\section{Author details \\ ${ }^{1}$ Department of Medicine, Division of Hematology, Cancer Institute, and Insti- tute for Stem Cell Biology and Regenerative Medicine, Stanford University, Stanford, CA, USA. ${ }^{2}$ Stanford University School of Medicine, Stanford, CA, USA. ${ }^{3}$ Department of Medicine, Stanford University, Stanford, CA, USA. ${ }^{4}$ Department of Pathology, Stanford University, Stanford, CA, USA. ${ }^{5}$ Depart- ment of Medicine, Division of Blood and Marrow Transplantation, Stanford University, Stanford, CA, USA. ${ }^{6}$ Department of Medicine, Division of Infectious Diseases \& Geographic Medicine, Stanford University, Stanford, CA, USA.}

Received: 6 August 2020 Accepted: 15 February 2021

Published online: 28 March 2021 


\section{References}

1. Liu L, Zhang X, Feng S. Epstein-Barr virus-related post-transplantation lymphoproliferative disorders after allogeneic hematopoietic stem cell transplantation. Biol Blood Marrow Transplant. 2018;24(7):1341-9.

2. Rasche L, Kapp M, Einsele H, Mielke S. EBV-induced post transplant lymphoproliferative disorders: a persisting challenge in allogeneic hematopoetic SCT. Bone Marrow Transplant. 2014;49(2):163-7.

3. Romero S, Montoro J, Guinot M, Almenar L, Andreu R, Balaguer A, et al. Post-transplant lymphoproliferative disorders after solid organ and hematopoietic stem cell transplantation. Leuk Lymphoma. 2019;60(1):142-50.

4. Styczynski J, Gil L, Tridello G, Ljungman P, Donnelly JP, van der Velden W, et al. Response to rituximab-based therapy and risk factor analysis in Epstein Barr virus-related lymphoproliferative disorder after hematopoietic stem cell transplant in children and adults: a study from the infectious diseases working party of the European group for blood and marrow transplantation. Clin Infect Dis. 2013;57(6):794-802.

5. Curtis RE, Travis LB, Rowlings PA, Socié G, Kingma DW, Banks PM, et al. Risk of lymphoproliferative disorders after bone marrow transplantation: a multi-institutional study. Blood. 1999:94(7):2208-16.

6. Landgren O, Gilbert ES, Rizzo JD, Socié G, Banks PM, Sobocinski KA, et al. Risk factors for lymphoproliferative disorders after allogeneic hematopoietic cell transplantation. Blood. 2009;113(20):4992-5001.

7. Hou H-A, Yao M, Tang J-L, Chen Y-K, Ko B-S, Huang S-Y, et al. Poor outcome in post transplant lymphoproliferative disorder with pulmonary involvement after allogeneic hematopoietic SCT: 13 years' experience in a single institute. Bone Marrow Transplant. 2009:43(4):315-21.

8. Buyck HCE, Ball S, Junagade P, Marsh J, Chakrabarti S. Prior immunosuppressive therapy with antithymocyte globulin increases the risk of EBV-related lymphoproliferative disorder following allo-SCT for acquired aplastic anaemia. Bone Marrow Transplant. 2009:43(10):813-6.

9. Ocheni S, Kroeger N, Zabelina T, Sobottka I, Ayuk F, Wolschke C, et al. EBV reactivation and post transplant lymphoproliferative disorders following allogeneic SCT. Bone Marrow Transplant. 2008;42(3):181-6.
10. Styczynski J, van der Velden W, Fox CP, Engelhard D, de la Camara R, Cordonnier C, et al. Management of Epstein-Barr virus infections and post-transplant lymphoproliferative disorders in patients after allogeneic hematopoietic stem cell transplantation Sixth European Conference on Infections in Leukemia (ECIL-6) guidelines. Haematologica. 2016;101(7):803-11.

11. Liu Q, Xuan L, Liu H, Huang F, Zhou H, Fan Z, et al. Molecular monitoring and stepwise preemptive therapy for Epstein-Barr virus viremia after allogeneic stem cell transplantation. Am J Hematol. 2013;88(7):550-5.

12. Kunitomi A, Arima N, Ishikawa T. Epstein-barr virus-associated post-transplant lymphoproliferative disorders presented as interstitial pneumonia successful recovery with rituximab. Haematologica. 2007;92(4):e49-52.

13. Panoskaltsis-Mortari A, Griese M, Madtes DK, Belperio JA, Haddad IY, Folz RJ, et al. An official American thoracic society research statement: noninfectious lung injury after hematopoietic stem cell transplantation: idiopathic pneumonia syndrome. Am J Respir Crit Care Med. 2011;183(9):1262-79.

14. Burney K, Bradley M, Buckley A, Lyburn I, Rye A, Hopkins R. Posttransplant lymphoproliferative disorder: a pictorial review. Australas Radiol. 2006:50(5):412-8.

15. Sauter A, Faul C, Bitzer M, Bares R, Kraus S, Fenchel M, et al. Imaging findings in immunosuppressed patients with Epstein Barr virus-related B cell malignant lymphoma. Am J Roentgenol. 2010;194(2):W141-9.

16. Pina-Oviedo S, Miranda RN, Medeiros LJ. Cancer therapy-associated lymphoproliferative disorders: an under-recognized type of immunodeficiency-associated lymphoproliferative disorder. Am J Surg Pathol. 2017. https://doi.org/10.1097/PAS.0000000000000954.

\section{Publisher's Note}

Springer Nature remains neutral with regard to jurisdictional claims in published maps and institutional affiliations.
Ready to submit your research? Choose BMC and benefit from:

- fast, convenient online submission

- thorough peer review by experienced researchers in your field

- rapid publication on acceptance

- support for research data, including large and complex data types

- gold Open Access which fosters wider collaboration and increased citations

- maximum visibility for your research: over $100 \mathrm{M}$ website views per year

At BMC, research is always in progress.

Learn more biomedcentral.com/submissions 